\title{
Decision Support in Uncertain Environments
}

\author{
Hany M. S. Lala, Kamal A. ElDahshan \\ Al-Azhar University, Cairo, Egypt
}

\begin{abstract}
There are ambiguities and vagueness in solar radiation records during a day. As a consequence, the development and adaptation of automatic knowledge acquisition techniques under uncertainty is entirely advisable. Among them fuzzy sets theory formulizes and analyses the situations in which the uncertainty is due to non-precise environment. Any good decision is based on information. Decision makers should reduce their uncertainty by obtaining as much reliable and consistent information as possible. In this paper, we introduce a novel decision support system for handling uncertain climate data consolidated with SAS application system. This novel approach is based on the membership function and multidimensional analysis. From our findings, we conclude that data warehousing and data mining are essentials for an effective decision support system.
\end{abstract}

\section{Introduction}

Because organizations frequently process uncertain information, decision makers should reduce their uncertainty by obtaining as much reliable and consistent information as possible. Due to the potential benefits of a data warehouse as well as internal and external pressure for creating a competitive advantage, many organizations have launched data warehouse projects with the expectations of acquiring a consistent and reliable source of data for their DSS. Therefore, a DSS with data warehouse should improve the performance of users by improving information accessibility which positively affects the quality of decision making [1].

At present there is a great need to provide decision makers from middle management upward with information at the correct level of detail to support decision making. Data warehousing, on-line analytical processing (OLAP), and data mining provide this functionality [2].

Although there are researchers in fields of certainty, such as the natural science fields of physics, mathematics, biology, and in the social science fields of philosophy, economy, society, psychology, cognition, almost nobody places suspicion on the uncertain essence of the world. An increasing number of scientists believe that uncertainty is the charm of the world and only uncertainty itself is certain!

For a long time, humans thought uncertainty was equal to randomness. Probability theory is the main mathematical tool to solve the problem of randomness. With more in-depth studies, people found a kind of uncertainties that could not be described with randomness. That was "fuzziness". Fuzziness is a characteristic feature of modern science to describe quantitative relationships and space formation by using precise definitions and rigidly proven theorems, and to explore the laws of the objective world by using precisely controlled experimental methods, accurate measurements, and calculation so as to establish a rigorous theoretic system [3].

There are ambiguities and vagueness in solar radiation records during a day. There is a desired need for providing a simple technique whereby uncertainties in the process of solar radiation measurements being handled.

In this paper, we introduce a novel decision support system for handling uncertain climate data consolidated with SAS application system. This novel approach is based on the membership function and multidimensional analysis.

The rest of the paper is organized as follows. The next section reviews related work. In Section 3 we will describe the characteristics of decision support systems. In Section 4 we demonstrate the basic data warehousing concepts. Section 5 provides the definition of a general framework for fuzzy sets in the presence of uncertainty. Section 6 introduces climate case study. In Section 7 we present our approach for solving this problem. Section 8 presents the results analysis. Section 9 shows 
the results that we have obtained when we employed SAS application system. Finally, we conclude with a discussion of the results.

\section{Related work}

Many different formal techniques, both numerical and symbolic, have been developed over the past three decades for dealing with incomplete and uncertain information. Hunter and Parsons in [4] reviewed some of the most important of these formalisms, describing how they work, and in what ways they differ from one another. Hernandez and Recasens in [5] provided the definition of a general framework for the induction of decision trees in the presence of uncertainty.

The management of uncertainty in database systems is a very important problem as the information is often vague. Urrutia et al proposed a FuzzyEER in [6] which is an extension of the EER model in order to manage uncertainty in fuzzy databases.

Hoda et al introduced in [7] a fuzzy decision support system (FDSS) with a new decision making structure, which can be applied to manage the crisis conditions in any large scale systems with many parameters.

D. Burdick et al in [8] extended the OLAP data model to represent data ambiguity, specifically imprecision and uncertainty, and introduced an allocation-based approach to the semantics of aggregation queries over such data. While there was much work on representing and querying ambiguous data, to their knowledge this was the first paper to handle both imprecision and uncertainty in an OLAP setting.

In this context, we introduce a novel decision support system for handling uncertain climate data, using the data warehousing technology combined with fuzzy sets theory as a mathematical data mining technique in the presence of uncertainty, consolidated with SAS application system.

\section{Decision support systems}

Because of the importance of high-quality decision making, firms are investing heavily in decision support systems (DSS), which consist of technologies and applications designed to help users make better business decisions.

The term DSS can be used as an umbrella term to describe any computerized system that supports decision making in an organization [9].

New tools such as OLAP, data warehousing, data mining added promised capabilities and easy access tools for computer-aided decision making. DSS accompanied with such tools provide firms with the capability to amass information, develop knowledge about operations, and change decision-making behavior to achieve profitability and other business goals.

According to [10], "A decision support system is a computer-based information system whose primary purpose is to provide knowledge workers with information on which to base informed decisions".

Decision support systems, by definition, exist to help people make decisions. They do not make decisions by themselves. This is why they are called decision support systems, not decision-making systems.

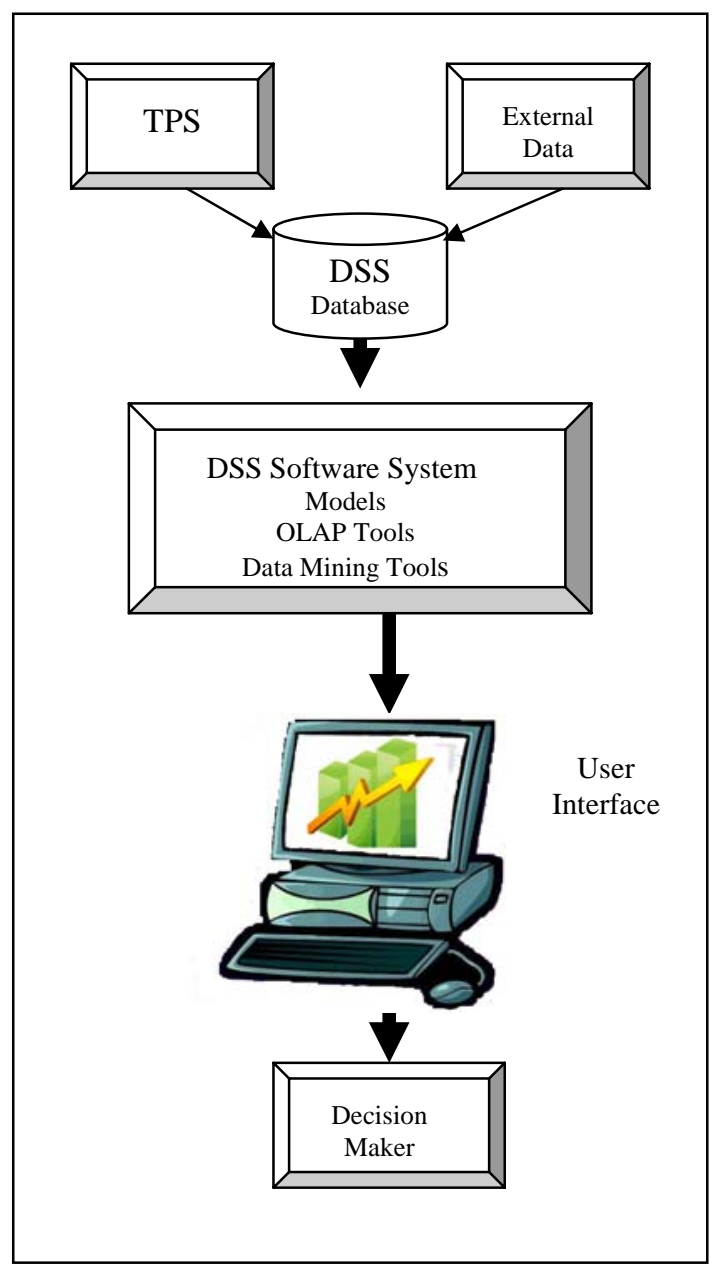

Figure 1. A schematic view of DSS 
DSS include a database used for query and analysis; a software system; and a user interface as shown in Figure 1 [11].

\section{Data warehousing}

As the need to make quick decisions has gained importance, the data available to support the decisionmaking process has proliferated. The overwhelming market acceptance of relational databases, the internet as a data source, and the availability of inexpensive platforms combine to allow corporations to accumulate and/or access a massive amount of data [12].

According to W. H. Inmon "A data warehouse is a subject-oriented, integrated, time-variant, and nonvolatile collection of data in support of management's decision making process" [13].

A data warehouse can be viewed as a data repository for an organization set up to support strategic decisionmaking. They store operational data in a separate system that a manager can freely access without interrupting the operational systems. The data in being transferred is prepared to ensure its quality and also the data warehouses are often set up to store historical data.

The most popular data model for a data warehouse is a multidimensional model. Such a model can exist in the form of a star schema, a snowflake schema, or a fact constellation schema.

In the multidimensional model, data are organized into multiple dimensions, and each dimension contains multiple levels of abstraction defined by concept hierarchies. This organization provides users with the flexibility to view data from different perspectives.

In the last years data warehouses have been recognized by a large number of organizations as a solution for exploiting the large quantity of information stored in their operational systems and for improving their decisional processes [14].

\section{Fuzzy sets}

In 1965, system theorist Lotfi A. Zadeh, who is called "father of fuzzy logic" published Fuzzy Sets where a more flexible sense of membership is possible. Fuzzy sets theory formulizes and analyses the situations in which the uncertainty is due to the non-precise (vague/fuzzy) data, or, in general, non-precise environment [15].

\section{Definition}

Let $U$ be the universal set. Any mapping from $U$ to the real interval $[0,1]$

$$
\begin{gathered}
\mu_{\tilde{\mathrm{A}}}: U \rightarrow[0,1] \\
\forall x \in U, x \rightarrow \mu_{\tilde{\mathrm{A}}}(x)
\end{gathered}
$$

determines a fuzzy set $\tilde{A}$ on $U$. $\mu_{\tilde{A}}$ is the membership function of $\tilde{A}$, and $\mu_{\tilde{A}}(\mathrm{x})$ is the membership degree of $\mathrm{x}$ for $\tilde{A}$. The fuzzy set $\tilde{A}$ divides $U$ into three parts: Kernel consists of the elements that belong to Ã totally, external consists of all the elements that do not belong to $\tilde{\mathrm{A}}$ and boundary consists of the elements that partly belong to $\tilde{A}[16]$.

The foundational problem of fuzzy sets is how to confirm a certain membership function. Demanding precise specifications would contradict the whole concept of fuzzy thinking.

However, there is no strict method for that. There are six types of functions: Linear, Gamma, Concave/convex, Cauchy, Mountain-shaped and bell [17].

\section{Case study}

Knowledge of global solar radiation is essential in the prediction, study and design of the economic viability of systems which use solar energy. Information on global solar radiation received at any site (preferably gained over a long period) should be useful not only to the locality where the radiation data is collected but also for the wider world community [18].

A global study of the world distribution of global solar radiation requires knowledge of the radiation data in various countries and for the purpose of world wide marketing, the designers and manufactures of solar equipment will need to know the average of global solar radiation available in different and specific regions [19].

Long-term variations in solar radiation at Earth's surface can affect our climate, the hydrological cycle, snow melt, plant photosynthesis, evaporation, crop growth, and solar power. Solar radiation is also a variable needed for biophysical models to evaluate risk of forest fires, hydrological simulation models and mathematical models of natural processes. Hence, in many occasions, a record of observed solar radiation or an estimate of radiation is required.

Several researchers have reported a widespread decrease in the amount of solar radiation received at the earth's surface during the second half of the $20^{\text {th }}$ 
century, a phenomenon that has come to be known as solar dimming [20].

The aim of this study is to measure the accuracy of global radiation measurements using our proposed decision support system. The assessment processes is based on surface observations for global radiation over seven sites in Egypt. This monthly climatologically data represents different climatic regions of Egypt ranged from Aswan (latitude $23^{\circ} 5^{\prime}$ ) to Matruh (latitude $31^{\circ} 20^{\prime}$ ), and was registered between the years 1973 and 1996.

In this case study, the subject area would be global solar radiation. The dimensions of analysis would be Region and Time. The requirement is to analyze global solar radiation according to region and time. Time is regarded as a necessary dimension of analysis (time variance is a characteristic of data warehouses) and so is always included as one of the dimensions of analysis. We use the star schema as a multidimensional model for building the data warehouse as shown in Figure 2.

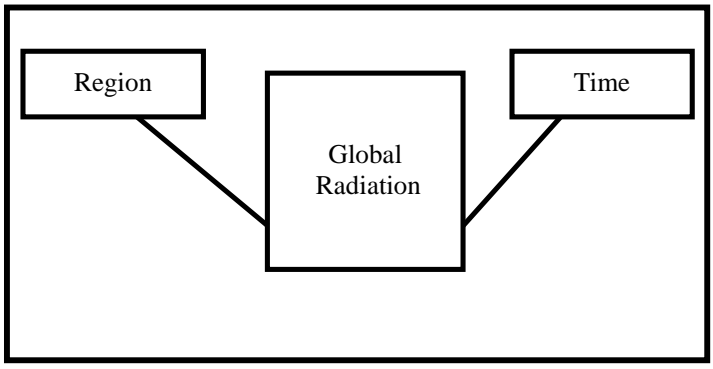

Figure 2. The star schema for climate study

\section{Multidimensional Least Square Membership Algorithm}

In this work, we introduce a novel decision support system for handling uncertain climate data using the data warehousing technology combined with fuzzy sets theory as a mathematical data mining technique in the presence of uncertainty. Figure 3 illustrates our DSS functionalities. We do this by determining the membership function for "global radiation" property that represents uncertainty problem in the data set applying a multidimensional analysis. The main technology that we have used for all technical work in this research was Oracle Application Development Framework (ADF) business components combined with Oracle JDeveloper.
Notation:

$\mathrm{k}$ is calculated so that the error sum of squares $S S E$ is minimized.

$$
\begin{aligned}
& S S E=\sum\left(y-\mu_{\tilde{\mathrm{A}}}(x)\right)^{2} \\
& S S T=\sum(y-\bar{y})^{2} \\
& S S R=\sum\left(\mu_{\tilde{\mathrm{A}}}(x)-\bar{y}\right)^{2} \\
& \text { Finally, } \quad r^{2}=\frac{S S R}{S S T},
\end{aligned}
$$

which represents the coefficient of determination.

Input:

i) Data with uncertainty problem for some properties “Ai".

(Assuming that xi's are Ai's values and their corresponding validating ratio yi's).

ii)The dimensions of analysis.

Output:

i)The most appropriate membership function for each property "Ai”.

ii)The preferred dimension of analysis.

Procedure:

For each property “Ai” Do

For each dimension of analysis Do

Begin

Step1:

Apply model (1): Calculate the following:

$$
k_{i}=\frac{\sum x-\sum x y}{\sum x^{2}}, S S E_{i 1}, r_{i 1}^{2}=\frac{S S R_{i 1}}{S S T_{i 1}}
$$

Step2:

Apply model (2):

$\mathrm{Y}=1+\ln y$ 


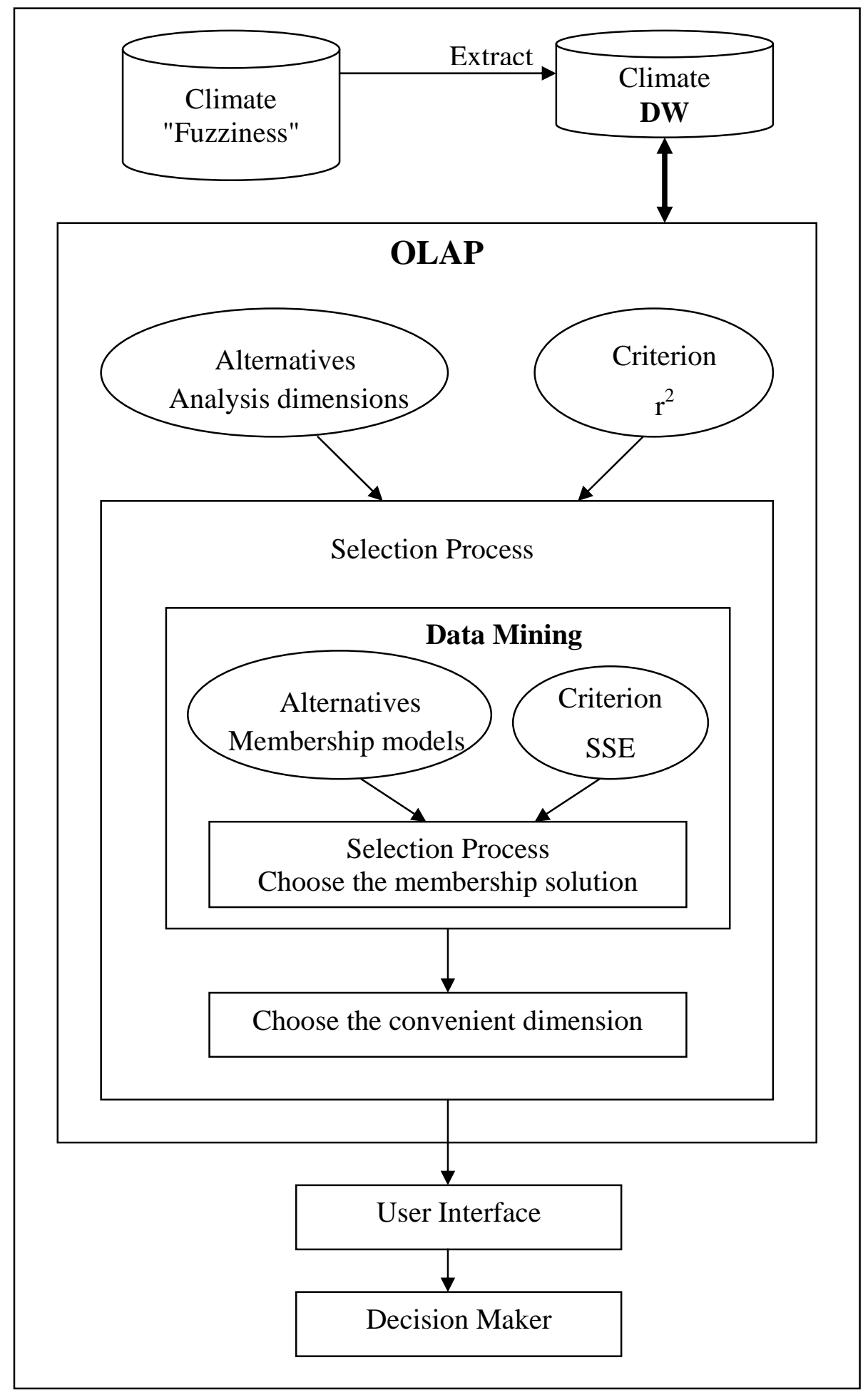

Figure 3. The proposed DSS functionalities 
Calculate:

$$
\begin{aligned}
& k_{i}=\frac{\sum x-\sum x Y}{\sum x^{2}} \\
& , S S E_{i 2}, r_{i 2}^{2}=\frac{S S R_{i 2}}{S S T_{i 2}}
\end{aligned}
$$

Step3:

Apply model (3):

Make the following transformations $X=\ln x$

$Y=\ln (1-y)$

Determine: $\quad x_{0} \rightarrow \mu_{\tilde{A}_{i}}\left(x_{0}\right)=0$

Calculate:

$$
\begin{gathered}
k_{i}=\frac{\sum X Y}{\sum X^{2}-\ln \left(x_{0}\right) \sum X} \\
S_{i 3}, r_{i 3}^{2}=\frac{S S R_{i 3}}{S S T_{i 3}}
\end{gathered}
$$

Step 4:

Apply model (4):

$$
\begin{aligned}
& X=-x^{2} \\
& Y=\frac{1}{y}
\end{aligned}
$$

Calculate:

$k_{i}=\frac{\sum X-\sum X Y}{\sum X^{2}}$

$S_{i 4}, r_{i 4}^{2}=\frac{S S R_{i 4}}{S S T_{i 4}}$

Step 5:

Apply model (5):

$$
Y=-\frac{2}{\pi} \sin ^{-1}(1-2 y)
$$

Calculate:

$$
\begin{gathered}
k_{i}=\frac{\sum x-\sum x Y}{\sum x^{2}} \\
S_{i 5}, r_{i 5}^{2}=\frac{S S R_{i 5}}{S S T_{i 5}}
\end{gathered}
$$

Step 6:

Apply model (6):
Determine:

$$
\begin{aligned}
& x_{0} \rightarrow \mu_{\tilde{A_{i}}}\left(x_{0}\right)=1 \\
& Y=1-\sqrt{\ln \left(\frac{1}{y}\right)} \\
& X=x-x_{0}
\end{aligned}
$$

Calculate:

$$
k_{i}=\frac{\sum X-\sum X Y}{\sum X^{2}}
$$$$
\text { , SSE }{ }_{i 6}, r_{i 6}^{2}=\frac{S S R_{i 6}}{S S T_{i 6}}
$$

Final Step:

Choose the membership function according to $\min _{j} \mathrm{SSE}_{i j}$

Choose the preferred dimension of analysis according to

$$
\max _{j} r_{i j}^{2}
$$

End

\section{Experimental results}

In this section we will introduce the results that we have obtained after applying our proposed algorithm for the climate case study.

\subsection{Multidimensional analysis}

We applied our methodology through the dimensions of analysis in distinct directions which were taken as a part of the input for our proposed algorithm.

\subsubsection{Region dimension}

Table 1 summarizes the patterns that have been detected when we applied our algorithm through region dimension.

We mention that "Gamma" is the best membership function from the proposed models that handles the uncertainty problem for global radiation measurements through region dimension. 
Table 1. The patterns detected through region dimension

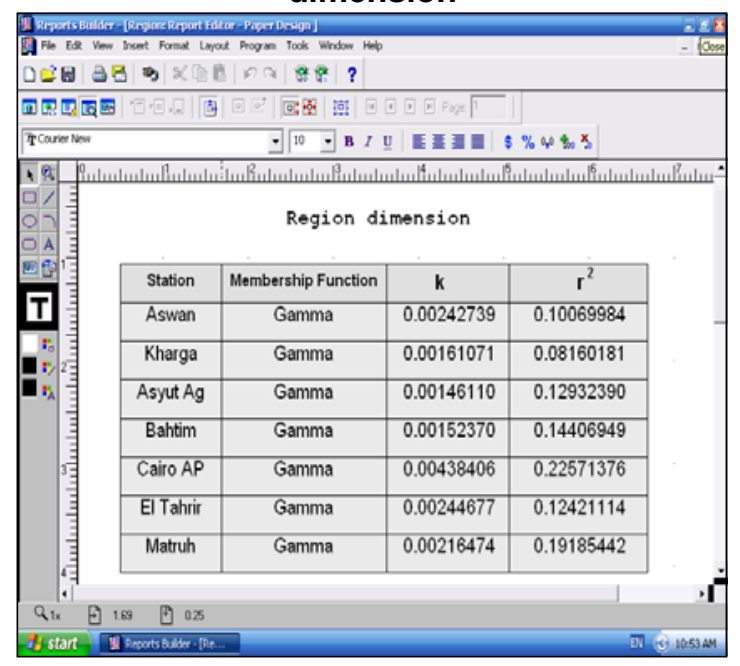

\subsubsection{Time dimension}

Table 2 summarizes the patterns that have been detected when we applied our algorithm through time dimension.

Table 2. The patterns detected through time dimension

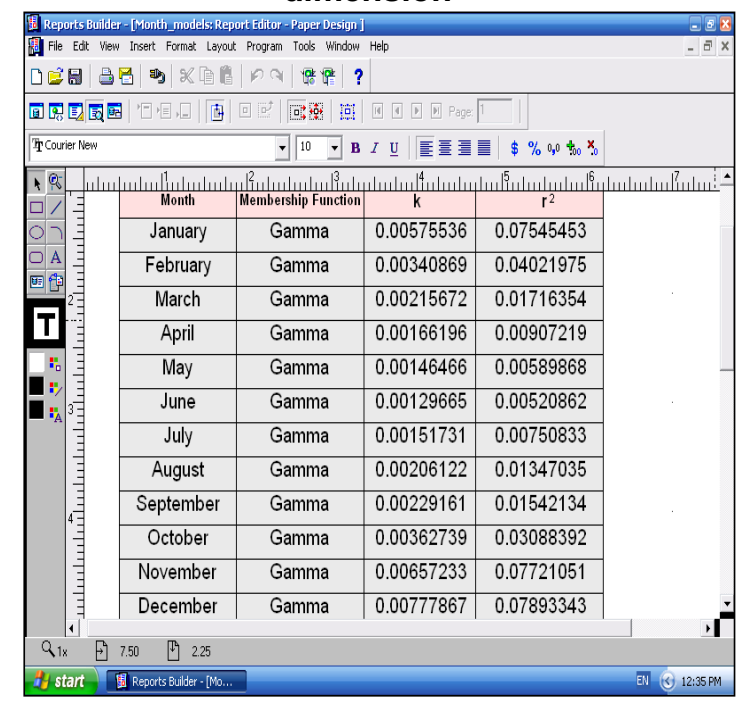

Also, we mention that "Gamma" is the best membership function from the proposed models that handles the uncertainty problem for global radiation measurements through time dimension.

\subsubsection{Region and time dimensions}

Table 3 and 4 summarize some of the patterns that have been detected when we applied our algorithm through region, and time dimensions together.

Table 3. The patterns detected in "Asyut Ag" station

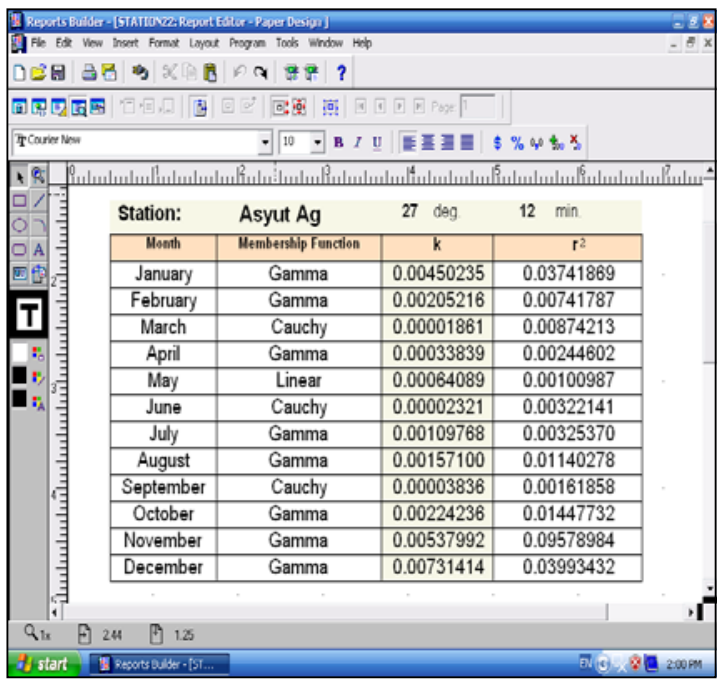

Table 4. The patterns detected in "El- Tahrir" station

\begin{tabular}{|c|c|c|c|c|c|}
\hline \multirow{2}{*}{\multicolumn{6}{|c|}{ 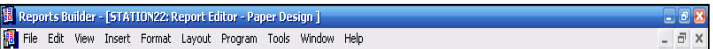 }} \\
\hline & & & & & $-a x$ \\
\hline \multicolumn{6}{|c|}{ 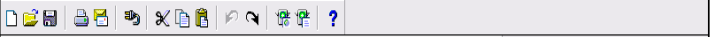 } \\
\hline \multicolumn{6}{|c|}{ 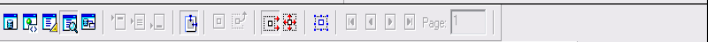 } \\
\hline Trcoulier New & & $\nabla 10$ в $I$ & 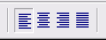 & \multicolumn{2}{|c|}{$\$ \% 0,0,500$} \\
\hline \multicolumn{6}{|c|}{ A } \\
\hline \multirow{3}{*}{ 要青 } & Station: & El Tahrir & 30 deg. & $39 \mathrm{~min}$. & \\
\hline & Month & Membership Function & k & $r^{2}$ & \\
\hline & January & Gamma & 0.00538166 & 0.03366818 & \\
\hline \multirow{2}{*}{ T学寻 } & February & Gamma & 0.00392198 & 0.01810657 & \\
\hline & March & Gamma & 0.00227421 & 0.01342998 & \\
\hline \multirow{2}{*}{ : } & April & Gamma & 0.00155552 & 0.00413420 & \\
\hline & May & Gamma & 0.00151612 & 0.01286811 & \\
\hline & June & Gamma & 0.00133147 & 0.00692097 & \\
\hline & July & Gamma & 0.00143108 & 0.00754950 & \\
\hline & August & Gamma & 0.00239298 & 0.01324166 & \\
\hline & September & Gammá & 0.00272294 & 0.00952699 & \\
\hline & October & Gamma & 0.00443712 & 0.02189766 & \\
\hline & November & Gamma & 0.00735689 & 0.02785622 & \\
\hline & December & Gamma & 0.00779000 & 0.028888665 & \\
\hline
\end{tabular}

Also, we mention that "Gamma" is the most convenient membership function from the proposed models that handles the uncertainty problem for global radiation measurements through region and time dimensions together. 


\subsection{Multidimensional decision}

The following table introduces a comparison statistics for the dimensions of analysis that we have studied the problem through according to the coefficient of determination (the scatter measure) which is denoted by $\mathrm{r}^{2}$.

Table 5. A comparison statistics for the dimensions of analysis according to $r^{2}$

\begin{tabular}{|l|c|c|c|}
\hline \multirow{2}{*}{$\begin{array}{l}\text { Dimensions } \\
\text { of analysis }\end{array}$} & \multicolumn{3}{|c|}{ coefficient of determination $\left(\mathrm{r}^{2}\right)$} \\
\cline { 2 - 4 } Time & 0.00520862 & 0.03137043 & 0.07893343 \\
\hline Region & 0.08160181 & 0.14249634 & 0.22571376 \\
\hline $\begin{array}{l}\text { Region and } \\
\text { time }\end{array}$ & 0.00096485 & 0.01983863 & 0.10313763 \\
\hline
\end{tabular}

From the previous results, it could be seen that the range of values for $r^{2}$ (the decision criterion) in the case of region dimension is better than those of the others. So, analyzing the data through region dimension is the best way for handling the uncertainty problem for global radiation measurements.

\subsection{Visual decision}

We end this section with the following chart that visualizes these membership functions whose Gamma behavior.

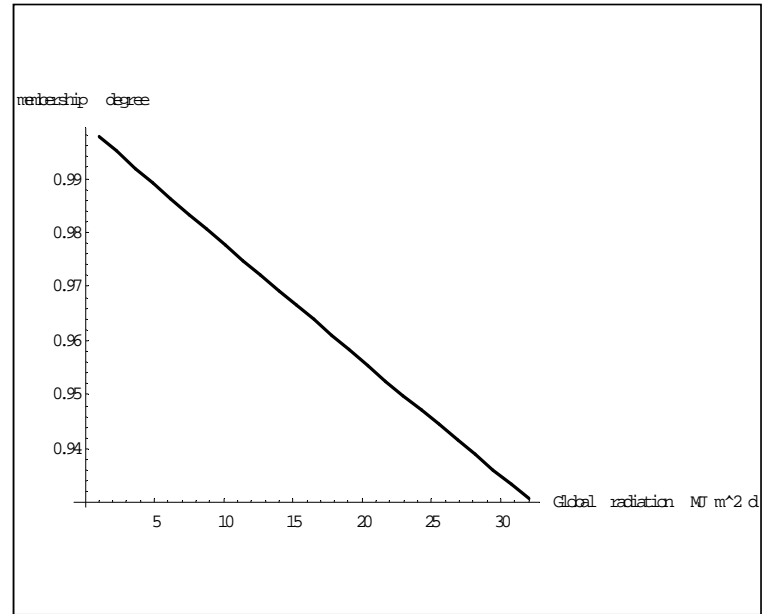

Figure 4. Gamma model in "Aswan" was the best through region dimension

From Figure 4 we mention that as we approach the kernel elements, the global radiation decreases. So, our experimental results confirm with the phenomenon that has come to be known as solar dimming.

\section{Utilizing SAS}

The following two figures show the results that we have obtained when we employed SAS application system, which is a ready-to-use DSS, as an external DSS layer.

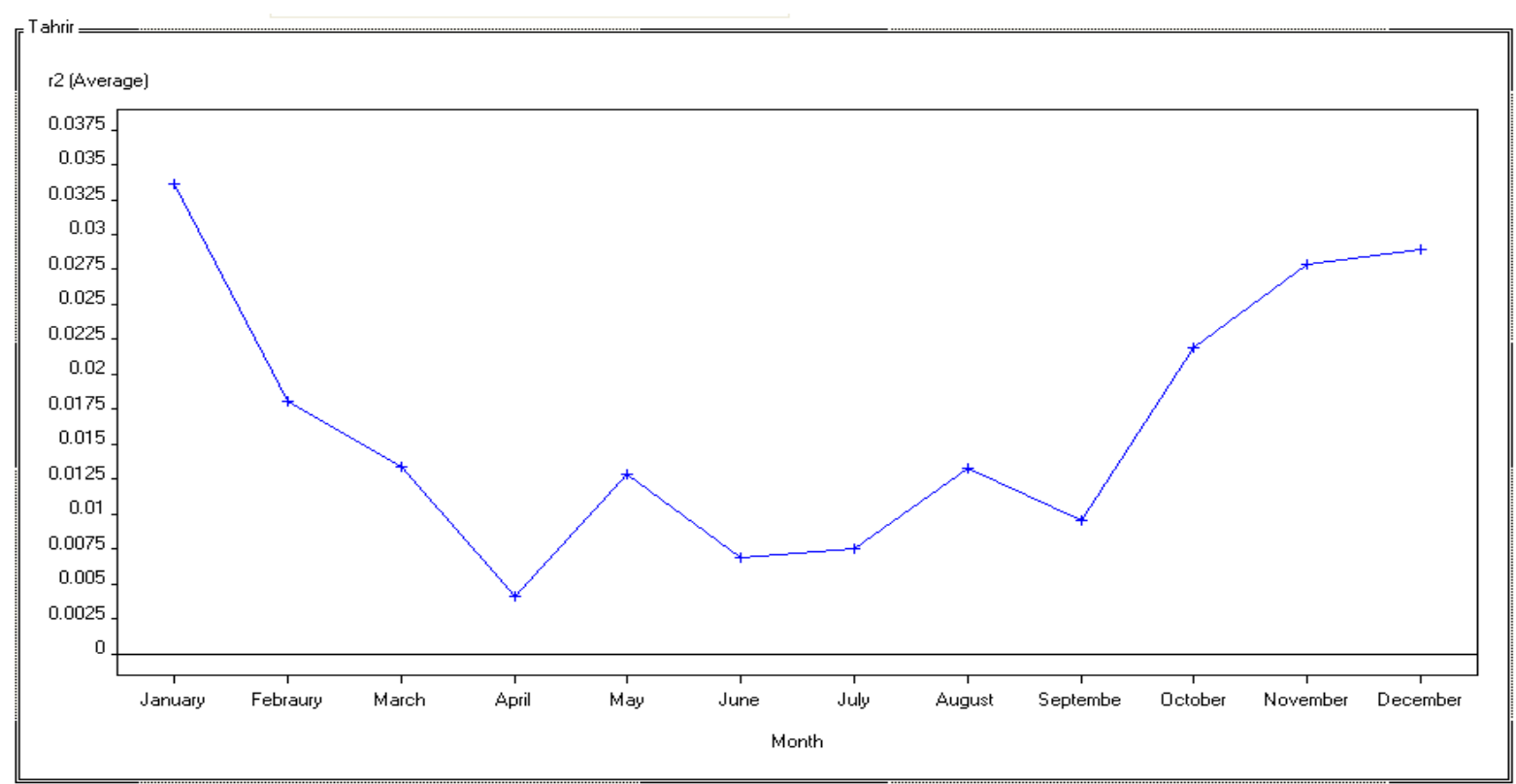

Figure 5. The correlation detected in "El- Tahrir" station through region and time dimension 


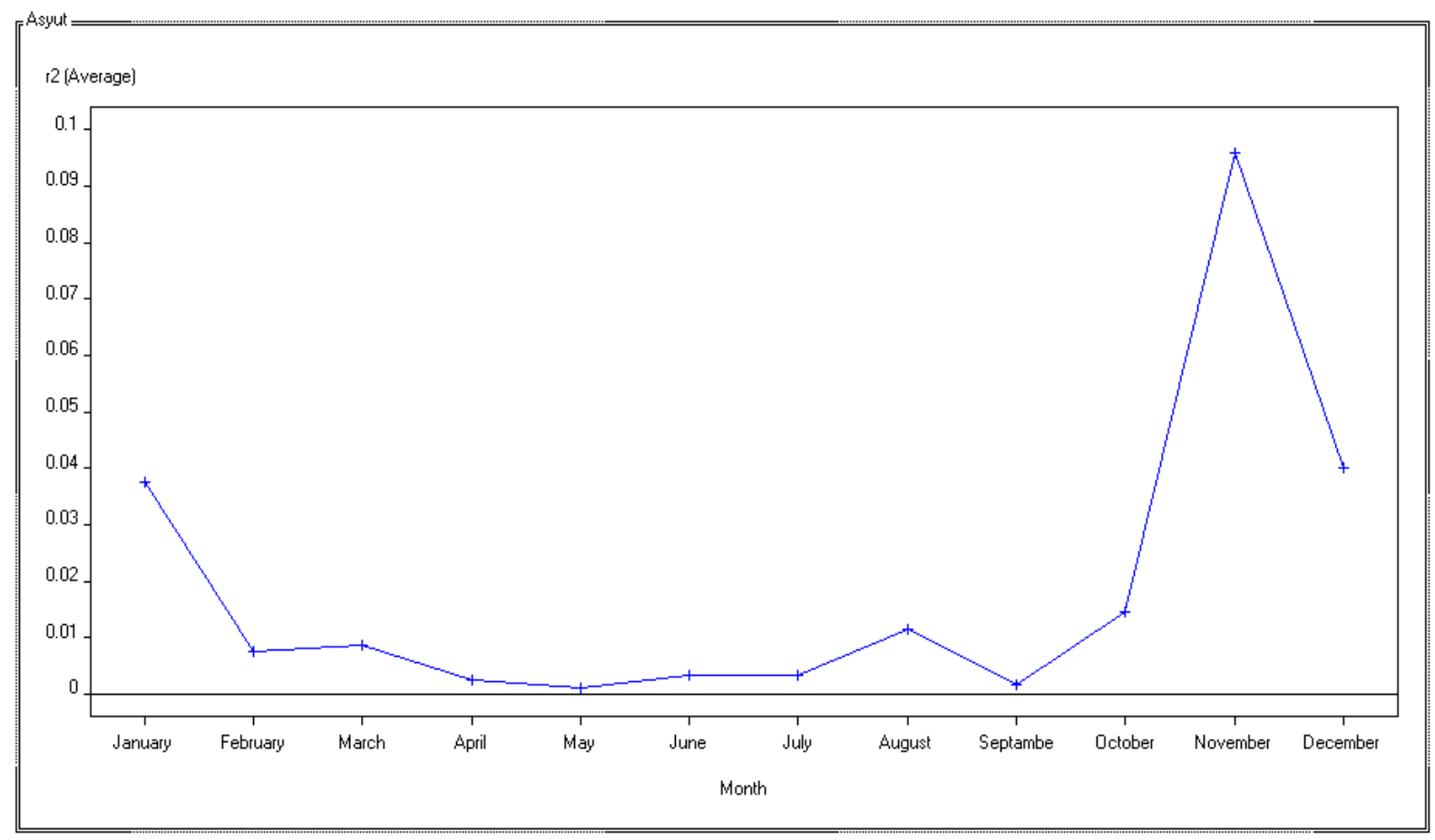

Figure 6. The correlation detected in "Asyut Ag" station through region and time dimension

Figure 5 and 6 show that the best correlation, when we applied SAS upon the detected patterns after implementing our algorithm through region and time dimensions together, was found in January for "ElTahrir" station and November for “Asyut Ag” respectively.

\section{Conclusion}

Because of the importance of high-quality decision making, firms are investing heavily in decision support systems, which consist of technologies and applications designed to help users make better business decisions. At present there is a great need to provide decision makers with information at the correct level of detail to support decision making. Data warehousing, OLAP, and data mining provide this functionality. The aim of this study was to simulate the uncertainty of global radiation measurements in order to help users make better decisions. This was handled by introducing a novel decision support system consolidated with SAS application system.

This novel approach was based on the membership function and multidimensional analysis. The assessment processes was based on surface observations for global radiation over seven sites in Egypt. From our findings, we conclude that analyzing the data through region dimension is the best way for handling the uncertainty problem for global radiation measurements. Also, we conclude that "Gamma" is the most convenient membership function among the proposed models. Also, we observe that as we approach the kernel elements, the global radiation decreases. So, our experimental results agree with the natural phenomenon of solar dimming.

\section{Acknowledgements}

We would like to thank our colleagues at the Astronomy and meteorology department, faculty of science, Al Azhar University who supported us with the case study data.

\section{References}

[1] Y.T. Park, "An empirical investigation of the effects of data warehousing on decision performance", Information \& Management, 43, 51-61, 2006.

[2] K. A. ElDahshan and H. M. Lala, "Data warehouse based statistical mining", AIML 9 (1), pp.41-48, 2009. 
[3] D. Li and Y. Du, Artificial Intelligence with Uncertainty, Taylor \& Francis Group, LLC, 2008.

[4] A. Hunter and S. Parsons, "A review of uncertainty handling formalisms", Applications of Uncertainty Formalisms, LNAI 1455, pp.8-37. Springer-Verlag, 1998.

[5] E. Hernandez and J. Recasens, "A general framework for induction of decision trees under uncertainty", Modelling with Words, LNAI 2873, pp.26-43, Springer-Verlag, 2003.

[6] A. Urrutia, J. Galindo, L. Jimenez, and M. Piattini, "The past and future of information systems: 1976-2006 and beyond", IFIP, Volume 214, pp.201-217, Springer, 2006.

[7] Hoda Nokhbatolfoghahaayee, Mohammad Bagher Menhaj, and Masoud Shafiee, "Fuzzy decision support system for crisis management with a new structure for decision making ", Expert Systems with Applications 37, pp. 3545-3552, Elsevier Ltd. 2010.

[8] D. Burdick, P. M. Deshpande, T. S. Jayram, R. Ramakrishnan, and S. Vaithyanathan, "OLAP over uncertain and imprecise data", VLDB 16:123-144, 2007.

[9] E. Turban, J. E. Aronson, T. Liang, and R. Sharda, Decision Support and Business Intelligence Systems, eighth edition. Prentice Hall, 2007.

[10] E. G. Mallach, Decision Support and Data Warehouse Systems, McGraw-Hill, 2000.

[11] K. C. Laudon and J. P. Laudon, Management Information Systems, ninth edition, Pearson Prentice Hall, 2006.

[12] R. A. Moeller, Distributed Data Warehousing using Web technology, How to Build a More Cost-Effective and Flexible Warehouse, AMACOM, 2001.

[13] W. H. Inmon, Building the Data Warehouse, Fourth Edition, Wiley, 2005.

[14] P. Naggar, L. Pontieri, M. Pupo, G. Terracina, and E. Virardi, "A model and a toolkit for supporting incremental data warehouse construction”, DEXA, pp.123 -132, Springer 2002.

[15] S.M. Taheri, "Statistics with non-precise data: A main approach in the future", International Conference on Statistics: Investment In The Future, September 2004.

[16] G. Chen and T. T. Pham, Introduction to Fuzzy Systems, Taylor \& Francis Group, LLC, 2006.

[17] K. A. ElDahshan and H. M. Lala, "Mining Uncertain Data Warehouse", The Fifth International Conference for Internet Technology and Secured Transactions (ICITST2010), London, UK, 2010.
[18] J. G. M. Massaquoi, "Global solar radiation in Sierra Leone (West Africa)", Solar and Wind Technology 5(3), pp. 281-283, 1988.

[19] S. M. A. Ibrahim, "Predicted and measured global solar radiation in Egypt", Solar Energy (35), pp. 185-188, 1985.

[20] S. Cohen, B. Liepert, and G. Stanhill, "Global dimming comes of age", Eos Trans. AGU, Volume 85, Issue 38, p.362362, 2004. 